\title{
Acercamiento a la percepción del estudiantado sobre el portafolio como técnica de evaluación en un sistema de educación a distancia
}

\author{
Marcela Sanabria Hernández ${ }^{1}$ \\ Adriana Salas Ruiz ${ }^{2}$ \\ 1. Máster en Evaluación de Programas y Proyectos de Desarrollo de la Universidad de Costa Rica. Máster en Psicopedagogía de la \\ Universidad de la Salle. Actualmente Encargada de Cátedra de Psicopedagogía de la UNED. msanabria@uned.ac.cr \\ 2. Licenciada en Psicología. Universidad de Costa Rica. Actualmente docente de la Cátedra de Psicopedagogía de la UNED \\ adrisalasruiz@yahoo.es
}

\author{
Recibido: 6 de Julio 2012 • Aceptado: 12 de Diciembre 2012 \\ RESUMEN
}

\begin{abstract}
En este artículo se presentan los resultados de un ejercicio investigativo que se realizó para el curso de Detección de Problemas de Aprendizaje de la Universidad Estatal a Distancia, con el objetivo de identificar el valor agregado que la técnica del portafolio brindaba a los y las estudiantes en su proceso de aprendizaje. Por otra parte, se ofrece el análisis de las respuestas del estudiantado sobre el uso del portafolio a la luz de la modalidad a distancia, de las características de la técnica misma y de las acciones de mejora que pueden implementar quienes administran dicho curso.
\end{abstract}

Palabra Clave: Educación a distancia, técnica del portafolio, técnica de evaluación, aprendizaje significativo.

\section{ABSTRACT}

This article displays the results of a research exercise that took place at the course Detection of Learning Problems from the Learning Distance University of Costa Rica. The objective of research is to identify the added value of the technique called "portfolio," which was part of the students' learning process. On the other hand, this document analyzes the students' answers on various aspects related to distance learning, the characteristics of the "portfolio", and possible actions to improve the course.

Key words: Distance learning education, portfolio technique, grading technique, meaningful learning.

\section{Introducción}

El interés por conocer la opinión del estudiantado sobre la técnica del portafolio, utilizada para el curso de Detección de Problemas de Aprendizaje de la Universidad Estatal a Distancia, conllevó a realizar una investigación exploratoria que permitiera identificar aquello elementos a favor o en contra del uso de la técnica, en función de posibles mejoras o cambios, que sirvan como una experiencia evaluativa.

Para lograr dicho objetivo, por un período de tres cuatrimestres, se solicitó a quienes matriculaban el curso de Detección, que brindaran su opinión sobre la técnica del portafolio, que había sido parte del ejercicio evaluativo del curso, 
básicamente en dos áreas: aspectos positivos y aspectos por mejorar.

Es a partir de lo propuesto por quienes realizaron dicho ejercicio que se ofrece un análisis de las respuestas para identificar si los y las estudiantes consideran que existe algún beneficio en el uso de la técnica para su aprendizaje que pudiera ser replicado en experiencias posteriores, así también que sirviera para la toma de decisiones de quienes administran el curso en función de la pertinencia del mismo o bien de acciones de mejora.

\section{La educación a distancia}

La educación a distancia puede ser concebida como una modalidad educativa cuya característica principal es proveer a los y las estudiantes los insumos necesarios para que puedan gestionar sus procesos de aprendizaje y regulen, a su ritmo, la oferta curricular que se les brinda. De esta manera, permite llevar a cabo una experiencia pedagógica, sobrellevando la brecha del tiempo y el espacio físico pues no se requiere la presencia del estudiantado ni del equipo docente en un mismo momento y en un mismo espacio para el acto educativo.

Tiene como característica principal proveer a los y las estudiantes los insumos necesarios para que puedan gestionar sus procesos de aprendizaje y regulen a su ritmo la oferta curricular que se les brinda.

Para Manrique (2004: 4-6) es importante destacar como elementos esenciales y positivos de la educación a distancia los siguientes aspectos:

- Desarrollo de estrategias afectivas y emocionales. El estudiantado debe ser capaz de identificar y apropiarse de su estilo de aprendizaje, esto conlleva a un desarrollo de autoconfianza en las capacidades para el cumplimiento de las tareas propuestas en su proceso educativo.

- Desarrollo de estrategias de autoplanificación. Cada estudiante debe gestionar su proceso de aprendizaje a partir de sus propias características, necesidades e intereses por eso, la importancia de contar con un plan de estudio realista y efectivo; con tareas tales como la determinación de espacios físicos idóneos para estudiar, horarios, recursos, estrategias, uso de tic entre otras.

- Desarrollo de estrategias de autorregulación. Este aspecto correlaciona las estrategias que favorecen los procesos de aprendizaje y permiten en logro satisfactorio de las tareas según la meta de aprendizaje.

- Desarrollo de estrategias de autoevaluación. Son tareas orientadas a que el estudiantado logre identificar sus fortalezas y aspectos para mejorar en su proceso de aprendizaje; principalmente enfocadas hacia a toma de decisiones y cambios oportunos de su forma de acercarse al estudio.

De esta forma, el éxito de la educación a distancia se encuentra en el papel activo, la responsabilidad y el interés que demuestren quienes participan en él.

Otra característica de la educación a distancia corresponde a todo el instrumental que se diseña y se hace llegar al estudiantado; en procura de que cuenten con la información idónea para su proceso de aprendizaje. De esta manera, es común el uso de guías didácticas, guías de orientación de los cursos, libros de texto, plataformas virtuales, material de índole audiovisual y otros recursos que guían, apoyan y sustentan el proceso de aprendizaje del estudiante.

En la participación de los actores, tanta importancia tiene el o la estudiante como sus docentes. Este último no queda excluido en el proceso de educación a distancia, por el contrario su papel cobra otro significado en la medida que, como un facilitador del aprendizaje, crea las instancias y situaciones que conlleven al estudiantado a desarrollar las habilidades y destrezas propias de la carrera o programa que cursan.

Una educación a distancia no implica una educación de menor calidad; y es desde esta perspectiva que la responsabilidad de la institución por brindar una oferta de calidad y orientar su trabajo hacia el logro de la excelencia de los 
aprendizajes deberá ser un pilar en todo su accionar. Para Ávila (2005), la calidad en la educación a distancia, sea cual sea la posición que se asuma, está asociada a cuestionamientos relacionados con la visión y la misión de las instituciones que ofrecen esta modalidad (P 4). A partir de lo expuesto anteriormente, la educación a distancia podría caracterizarse por lo siguiente:

- No es un requisito que docentes y estudiantes se encuentren en una ubicación física y temporal al mismo tiempo.

- Se incorporan nuevos recursos para facilitar los procesos de aprendizaje; eomo por ejemplo, las tecnologías de información y comunicación (TIC).

- Se fomenta el aprendizaje autónomo.

- Se apoya al estudiante con la modalidad de tutorías.

- Debe existir toda una organización institucional para facilitar los proceso logísticos que conlleven al buen desempeño administrativo de las instancias que ofrecen esta modalidad.

\section{Rol del estudiante}

En los procesos educativos se busca que el estudiantado pueda tener un papel dinámico y de reflexión constante sobre su propio proceso de formación; en la educación a distancia, esta no es la excepción y, por el contrario, se promueve un rol muy activo, autónomo y autorregulado. Para ello, los y las estudiantes deben estar motivados y ser responsables ante su propia gestión en el acto educativo.

\section{Rol del docente}

El rol del docente debe ser orientado hacia la función de un mediador del proceso de aprendizaje. En principio este mediador -también llamado tutor, asesor pedagógico o guía- tiene como objetivo contribuir con la formación del estudiantado a partir de la propuesta curricular donde se encuentre inmerso. Es así como en este rol docente, la figura del tutor o tutora va a tener una gran responsabilidad al momento de realizar las orientaciones que conlleven al logro de objetivos planteados para cada asignatura de la carrera que se estudia en la modalidad a distancia.

No obstante, este papel va más allá; tiene sus implicaciones en el acompañamiento, la asesoría, la elaboración de estrategias del trabajo individual y grupal de quienes matriculen los diferentes cursos. Se puede caer en el error de considerar al tutor, tutora o docente a distancia solo como quien instruye con una tarea más de índole administrativa o logística de los cursos. Pero el rol del docente es de gran importancia ya que se vuelve el vínculo entre la propuesta curricular y el estudiantado. Una cualidad que debe reconocerse en esta propuesta de tutor o tutora es la especialidad en el área de la materia o asignatura que coordina, pero, también, con un conocimiento en aspectos de índole pedagógica, didáctica y metodológica.

\section{La evaluación en la educación a distancia}

Para Ramírez y Flores (2009), la evaluación del aprendizaje debe concebirse como el proceso por medio del cual se determina el grado en el que el alumnado logra los objetivos de aprendizaje. Se puede entender, también, como una forma de determinar qué se ha logrado en un tiempo y espacios determinados; así como, los aspectos que requieren un refuerzo. "Es por ello que todo proceso de evaluación de aprendizaje debe partir siempre de un conjunto claro de objetivos de aprendizaje que se determinen para un curso determinado" (p.4).

En los procesos de educación a distancia la evaluación tanto formativa como sumativa debe llevarse a cabo de forma sistemática y constante, de tal forma que le permita al estudiantado y a quien esté a cargo de los cursos determinar el logro de objetivos y las acciones para favorecer el aprendizaje. Es así como la evaluación de los aprendizajes en una modalidad a distancia se convierte en una herramienta que le permite al estudiantado tener una mayor y mejor comprensión de sus propias competencias y el progreso en el proceso en el cual son calificados (Ryan et al, 2002, citados por Ramírez y Flores 2009: 4). 


\section{Técnicas cualitativas de evaluación en la educación a distancia}

Al pensar en la evaluación en la educación a distancia, puede hacerse referencia a aspectos de diferente índole, desde el planteamiento de instrumental más tradicional hasta lo cualitativo. Lo importante es tener claridad sobre el objetivo a cumplir en la aplicación del instrumento, el contexto en que se está brindando el curso, la mediación pedagógica, el conocimiento y el dominio del estudiantado en el uso de la técnica.

Como ejemplo las siguientes técnicas cualitativas:

- El proyecto: se solicita realizar un informe o proyecto de un área o tema específico. En él se pueden ofrecer casos, trabajo de campo y observaciones, entre otros aspectos.

- El portafolio: dicha técnica permite observar el avance en el dominio de contenidos a partir de las evidencias presentadas por el estudiantado. Es una técnica muy creativa, pero sistemática que involucra procesos de investigación, de análisis y síntesis con respecto a lo que se ha aprendido.

- Estudio de casos: a partir de una situación concreta se solicita definir acciones y realizar análisis diversos a la luz de las temáticas estudiadas.

- Mapa conceptual: que permite de forma visual establecer los procesos y vínculos que se han desarrollado para evidenciar el aprendizaje adquirido.

\section{La técnica del portafolio}

El portafolio es una herramienta didáctica cuyo objetivo es la recolección y registro de noticias, fotografías, reflexiones, bitácoras, publicaciones y otros documentos que se organizan de manera lógica y secuencial en torno a un tema o área de conocimiento particular, según una serie de objetivos específicos y que cuenta con un hilo conductor. Un portafolio puede ser elaborado con diversos fines, y constituye un medio que permite poner en evidencia el nivel de dominio o conocimiento alcanzado por un sujeto en un área determinada (Murillo, 2012).

Esta técnica surge de las disciplinas artísticas en las que se emplea con el objetivo de mostrar las competencias desarrolladas por el o la profesional, de acuerdo con su área de desempeño; así como sus mejores trabajos realizados. Aplicada a otras disciplinas, esta técnica ha sido incluida como una estrategia metodológica de aprendizaje y evaluación, que adquiere un auge particular en los últimos años, cuando, a partir de los nuevos enfoques educativos basados en el desarrollo de competencias y habilidades, se ha puesto particular énfasis en el saber hacer tanto como en el dominio teórico de ciertos temas (Barragán, 2005)

En el ámbito de evaluación, el portafolio se utiliza como medio alternativo de comprobación de conocimientos ante las pruebas tradicionales con enfoque memorístico. Se trata, pues, de un instrumento de evaluación de carácter longitudinal que da énfasis al proceso de aprendizaje en un período determinado y, no, a productos puntuales en momentos específicos. En relación con esto, el portafolio es un "recurso didáctico que promueve una evaluación formativa, permite al alumno demostrar la profundidad de su aprendizaje, brinda la oportunidad de ver y poseer los resultados de su aprendizaje, proporciona oportunidades para reflexionar, aumenta su motivación por aprender, aprenden a aprender y a asumir mayor responsabilidad de su proceso de aprendizaje y proporciona una oportunidad de evaluar la enseñanza" (Martínez, 2007: 133)

El portafolio se ha utilizado como estrategia de evaluación en ciencias de la salud (Vera y Canalejas, 2007; Canalejas, 2010), en el sector de educación (Pozo y García, 2006; Martínez, 2007), en el ámbito del aprendizaje de idiomas entre otras (García, 2005). En todos estos casos se ha demostrado su efectividad como estrategia de aprendizaje significativo, que promueve una mejor comprensión y dominio de los temas para los cuales se ha aplicado y como herramienta de promoción útil y efectiva a nivel de rendimiento académico. Si bien es cierto se han encontrado limitaciones en su aplicación tales como el hecho de ser una herramienta que requiere de tiempo 
y dedicación y que puede generar cierto grado de ansiedad en algunos momentos ante la incertidumbre de si se está realizando de manera adecuada, en términos generales, son más las ventajas que las desventajas de su aplicación.

Entre los alcances de esta técnica, tanto en lo formativo, en el ámbito profesional, como en el desarrollo integral del estudiante como sujeto que aprende, se puede citar el desarrollo de destrezas y habilidades para la búsqueda de información y organización de la misma, la posibilidad de crear a partir de un conocimiento base, y la posibilidad de involucrarse en forma directa con el proceso de aprendizaje (Danielson y Abrutyn, 1997). Por otra parte, el uso de esta técnica permite que el estudiantado logre realizar procesos de reflexión y análisis tanto sobre el contenido en estudio como de la relación individual y grupal del futuro profesional con la asignatura estudiada.

De igual manera, otra de las ventajas halladas en los estudios realizados en relación con el uso del portafolio, es que esta es una herramienta que le ayuda al estudiante a ser más organizado, promueve el trabajo continuo lo que facilita un mejor aprovechamiento de los contenidos, pone en evidencia el proceso de aprendizaje que ha desarrollado el alumno en un período de tiempo determinado, entre otros (de la Peña, Pastor y Selma, 2012)

\section{Estructura del portafolio}

Pese a su versatilidad, como toda técnica de evaluación y comprobación de aprendizajes, el portafolio posee una estructura básica que permite tanto a quien lo elabora como a quien lo evalúa orientarse en torno a los contenidos fundamentales. En ese sentido, Barbera (2005) define los siguientes aspectos como fundamentales para la elaboración de todo portafolio:

- guía o índice de contenidos;

- apartado introductorio donde se detallen intenciones y punto de partido;

- temas centrales que conforman el cuerpo del portafolio;

- apartado de cierre o conclusiones.
Aunado a estos aspectos, se debe incluir en el portafolio información relacionada con: la autoría y audiencia del portafolio, los contenidos por desarrollar, los objetivos y competencias, la estructura y organización concreta y los criterios de evaluación aplicables al instrumento. Teniendo como base dichos criterios, la Cátedra de Psicopedagogía de la UNED, encargada de la administración de la materia de detección de problemas de aprendizaje, propone en las Orientaciones para el Curso ${ }^{1}$, la siguiente información para la elaboración del portafolio:

Información general sobre la estrategia de evaluación del "Portafolio" (UNED, 2011)

La estrategia de evaluación del portafolio es utilizada como medio para conocer el avance, asimilación y las vivencias del educando. El portafolio es una modalidad de evaluación basada en dos tipos de requisitos fundamentales:

- Que los estudiantes puedan demostrar qué es lo que están entendiendo y aprendiendo, a través de la organización de sus trabajos.

- Que el aprendizaje pueda ser evaluado a través de una colección de documentos.

Tiene cuatro elementos esenciales: contenido, tiempo, estructura y participación de los y las estudiantes.

1. Contenido: el portafolio es una colección de productos realizados por los y las estudiantes durante un curso. Estos productos pueden ser escritos, reportes, fotografía, dibujos, pinturas, mapas, experimento o acciones, entre otros. El propósito de cualquier sistema de portafolio es demostrar, a través de estos registros de trabajo, qué es lo que ha entendido y aprendido.

1. Las Orientaciones para el Curso es un documento de carácter oficial que se entrega al estudiante al momento de la matrícula y en el cual se encuentran dadas todas las especificaciones relacionadas con el curso, incluyendo objetivos, metodología de evaluación, criterios de promoción, cronograma de fechas, entre otros. 
2. Tiempo: una diferencia entre el portafolio y las pruebas es que el portafolio puede demostrar los esfuerzos en un período extenso. Para evidenciar el progreso en el portafolio, debe existir más de un "producto final", que refleje los pasos dados a través del proceso completo.

3. Estructura: como cualquier colección con significado, el portafolio debe estar cuidadosamente organizado. La organización de los contenidos puede establecerse a partir de la siguiente guía:

- Tabla de contenidos

- Introducción que identifica al estudiante y explica qué se encontrará en la colección

- Propósito del portafolio

- Desarrollo de temas en donde debe incluir el tema y sus comentarios

- Cualquier tipo de materiales de apoyo que considere que fortalece la comprensión del tema expuesto.

- Citas bibliográficas que amplíen el tema

- Una vez que el estudiante ha generado su conocimiento es fundamental que proponga sus conclusiones; en donde elabora un cierre sobre el proceso de aprendizaje.

4. Participación de los estudiantes: la evaluación a través del portafolio ofrece una oportunidad única para que el estudiantado se involucre en su propia evaluación de diversas maneras.
- Existe un compromiso por seleccionar de forma clara y pertinente los materiales que serán incluidos.

- Cada estudiante está involucrado en el proceso de evaluar el trabajo en su portafolio.

- Ofrece la posibilidad de externar sus comentarios e ideas acerca de su aprendizaje y sus logros a través de ejercicios sistemáticos de reflexión.

Los temas sobre los cuales las y los estudiantes debían realizar el portafolio incluían la calidad de la atención de las NEE en instituciones educativas públicas o privadas, caracterización del desarrollo motor y cognitivo de niños, niñas y adolescentes con discapacidad sensorial, motora o psíquica; adecuaciones curriculares en casos concretos: el caso de Carlitos; el rol de la evaluación curricular en las dificultades en el aprendizaje; papel de las materias especiales en la atención e integración de los estudiantes con NEE: el caso de las artes plásticas, música y educación física; y Las tecnologías de la información y de la comunicación (TIC) en el campo de las NEE (UNED, 2001).

A partir de esta información se plantearon una serie de acciones que cada estudiante debe realizar para cumplir con el portafolio. Estas acciones implicaban elementos que permitían a cada estudiante orientar la elaboración de su trabajo. En términos generales se propuso que el portafolio contuviera:

\begin{tabular}{|c|c|c|c|c|}
\hline Aspecto & Comentarios & Complemento & Citas bibliográficas & Otros \\
\hline 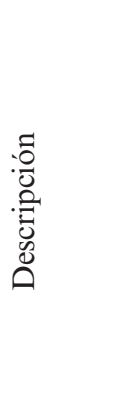 & $\begin{array}{l}\text { Presentan y analizan el } \\
\text { tema brindando opiniones } \\
\text { personales } \\
\text { Evidencian dominio de los } \\
\text { contenidos } \\
\text { Evidencian relación con } \\
\text { los otros apartados del } \\
\text { documento } \\
\text { Realizados con las propias } \\
\text { palabras de quien lo } \\
\text { elabora }\end{array}$ & $\begin{array}{l}\text { Documentos, artículos, } \\
\text { recortes, fotografías, } \\
\text { poesías, reflexiones u } \\
\text { otra información que } \\
\text { enriquecen la temática } \\
\text { en estudio }\end{array}$ & $\begin{array}{l}\text { Frases, párrafos } \\
\text { o contenido del } \\
\text { material del curso } \\
\text { que enriquecen } \\
\text { el desarrollo del } \\
\text { tema planteado, } \\
\text { especificando la } \\
\text { fuente de donde se } \\
\text { tomó la cita }\end{array}$ & $\begin{array}{l}\text { Actividades concretas } \\
\text { que incluyen una } \\
\text { guía o ficha de } \\
\text { trabajo específica }\end{array}$ \\
\hline
\end{tabular}

Fuente: Elaboración propia, 2012. 
Finalmente, se incluyó un apartado en el que los y las estudiantes, debían comentar acerca del desarrollo de la técnica tanto en aspectos positivos como en aquellos por mejorar.

\section{Propuesta de investigación}

\section{Contexto de la investigación}

La Cátedra de Psicopedagogía forma parte de la Escuela de Ciencias Sociales y Humanidades de la Universidad Estatal a Distancia. Dicha cátedra es la encargada de administrar el curso de Detección de problemas de Aprendizaje que se ofrece, como parte de la propuesta curricular de las carreras de Diplomado en I y II Ciclo y Diplomado en la Enseñanza del Francés, el curso de Detección de Problemas de Aprendizaje.

$\mathrm{La}$ asignatura tiene como propósito "proporcionar al estudiante una visión general e integrada de las dificultades de aprendizaje, entendida como necesidades educativas especiales, sus principales manifestaciones así como algunos planteamientos metodológicos estrategias que se deben seguir para que estos alumnos aprendan mejor" (UNED, 2001: 3).

De esta manera, el curso ha sido pensado para brindar a los futuros profesionales la teoría referida a temas como: conceptualización de los problemas de aprendizaje, detección de las necesidades educativas especiales y pautas generales de intervención para la educación inclusiva en el contexto educativo. Todo esto constituye un insumo que permite al estudiante crear y aplicar estrategias de intervención válidas y con respaldo teórico que le permitan tener una incidencia más efectiva como futuro profesional en su espacio de trabajo.

Con respecto a la evaluación, en el curso se ha trabajado con tres modalidades evaluativas:

- Elaboración de dos exámenes teóricos con un formato tradicional. Es decir, dos exámenes de comprobación de conocimientos de tipo memorístico, calendarizados, y que se llevan a cabo en alguna de las sedes de la UNED en una fecha previamente establecida.
- Un examen teórico con formato tradicional y un examen para elaborar en casa.

- Un examen teórico con formato tradicional (ya sea un ordinario de medio período o un comprensivo al final de cuatrimestre) $\mathrm{y}$ un portafolio.

Actualmente, la evaluación se maneja con este último formato (examen y portafolio).

\section{Justificación de la investigación}

El uso del portafolio como medio de evaluación en el curso de Detección de problemas de aprendizaje se implementa a partir del I cuatrimestre del 2009, como respuesta a la necesidad de un cambio metodológico a nivel de evaluación, que buscaba no sólo garantizar el dominio teórico de la materia sino promover una experiencia de aprendizaje significativo. Es por ello que surge la idea de aplicar un portafolio como parte de la evaluación que remplazara una de las dos pruebas escritas. Después de tres períodos de aplicación de esta nueva estrategia de evaluación, brota la inquietud a lo interno de la Cátedra por conocer la percepción del estudiantado en torno al portafolio como herramienta de evaluación.

De esta manera, como acercamiento inicial se propone realizar un sondeo entre los estudiantes por un período de tres cuatrimestres consecutivos, en el cual, se les solicitó a los y las estudiantes, que hicieran un comentario acerca del desarrollo de la técnica tanto en aspectos positivos como en aquellos por mejorar. Se indicó que la respuesta no requería mencionar información personal. La misma debía hacerse como un documento aparte que le permitiera a quien calificara los trabajos desprenderlo y analizar dicha información. También se señaló que no era un aspecto por calificar en el trabajo, pero era obligatorio el desarrollarla.

\section{Metodología}

Se trabajó con la totalidad de estudiantes matriculados en el curso Detección de Problemas de Aprendizaje en tres períodos de matrícula, durante el 2010 - 2011. Específicamente, se tomó en 
cuenta a las y los estudiantes matriculados en el último cuatrimestre del 2010 y dos primeros del 2011. Esto constituyó un total de 280 encuestas aplicadas e incluidas en el análisis de resultados.

Por su facilidad se seleccionó el uso de una encuesta autoadministrada que el estudiante debía entregar junto con su portafolio en el Centro Universitario de la UNED en el cual se encontraba empadronado.

La consigna dada a las y los estudiantes para la realización de la encuesta fue la siguiente: "Comente ampliamente sobre esta modalidad de evaluación llamada portafolio. Refiérase a su experiencia personal y de aprendizaje al realizarlo. Nos interesa saber su opinión. Esta pregunta desarróllela en página aparte para que el profesor pueda desprenderla" (UNED, 2001: 14).

Al final de cada cuatrimestre las encuestas se recogieron junto con los portafolios de las y los estudiantes. Posteriormente, la información fue sistematizada y organizada según la frecuencia de las respuestas brindadas por los alumnos.

\section{Resultados obtenidos}

Durante los tres cuatrimestres que se solicitó la información con respecto a la percepción del estudiantado sobre el uso de la técnica del portafolio, la misma fue recolectada y analizada. Para este análisis de clasificaron las opiniones en dos grandes áreas: los aspectos positivos y los aspectos por mejorar. Posterior a este ordenamiento de información, esta se jerarquiza en procura de establecer los criterios que se repetían con mayor frecuencia y aquellos que solo eran mencionados una única vez.

Las respuestas son ordenadas por categorías que permiten conglomerar la mayoría de respuestas en un solo sentido de interpretación; no obstante algunas de ellas no podían ser incorporadas en estas categorías y se reflejan como únicas en el cuadro que resume dicha información. Dichas respuestas fueron sistematizadas e integradas en un cuadro (anexo 1), en general reflejan una actitud positiva del estudiantado con respecto a la técnica del portafolio.

En términos globales, $12,5 \%$ de estudiantes consultados indican que la técnica del portafolio les permitió implementar procesos de planificación, ordenamiento, selección, recopilación, comparación y discriminación de información; de tal forma, que el trabajo por realizar evidenciara el dominio de los contenidos visto en el curso y la construcción de conocimientos presentada en la técnica utilizada para evaluar.

Para un 10,35\% de los y las estudiantes la realización del portafolio conllevó un ejercicio de reflexión crítica y de análisis, en cuanto a la información que se debía aportar; pero también, a la importancia de la temática para su formación y de acciones de mejora que deberían estarse dando desde los centros educativos.

Como se mencionó en apartados anteriores la evaluación de los aprendizajes en una modalidad a distancia es de gran importancia, porque le debe permitir tanto al estudiantado como la institución educativa identificar los conocimientos adquiridos y los cambios pertinentes en pro de favorecer un aprendizaje significativo. En ese sentido un $9,6 \%$ de los estudiantes mencionaron que la técnica del portafolio es una excelente forma de evaluar porque permite el ejercicio de llevar a la práctica los conocimientos adquiridos durante el curso.

El fomento a la lectura fue otro de los aspectos indicados como positivos por un $7,5 \%$ de los y las estudiantes, haciendo alusión a la exigencia de ir más allá de lo que el curso les ofrece en sus recursos o materiales. Para una mayor comprensión del sentir del estudiantado, todas las respuestas brindadas por ellos (as) se organizaron a partir de aspectos o temáticas específicas donde se reflejan las principales virtudes que se atribuyen al portafolio como técnica de evaluación: 


\begin{tabular}{|c|c|c|c|c|}
\hline Actitud: & $\begin{array}{l}\text { Aprendizaje } \\
\text { significativo }\end{array}$ & Creatividad & Evaluación & Investigación \\
\hline $\begin{array}{l}\text { Favorecen el compromiso } \\
\text { de los y las estudiantes. }\end{array}$ & $\begin{array}{l}\text { Favorece la lectura, } \\
\text { la criticidad. }\end{array}$ & \multirow[t]{3}{*}{$\begin{array}{l}\text { Favorece la } \\
\text { individualidad y } \\
\text { la innovación. }\end{array}$} & $\begin{array}{l}\text { Es una técnica } \\
\text { más valiosa que un } \\
\text { examen. }\end{array}$ & \multirow{3}{*}{$\begin{array}{l}\text { Requiere buscar } \\
\text { información más } \\
\text { allá de lo que } \\
\text { plantea el libro. } \\
\text { Permite } \\
\text { interrelacionar } \\
\text { temáticas. }\end{array}$} \\
\hline $\begin{array}{l}\text { Interés por realizar el } \\
\text { portafolio.Le permite }\end{array}$ & \multirow{2}{*}{$\begin{array}{l}\text { Permite la } \\
\text { actualización. } \\
\text { Es una técnica } \\
\text { constructivista. }\end{array}$} & & Evidencia los logros. & \\
\hline $\begin{array}{l}\text { al estudiantado asumir } \\
\text { una posición sobre los } \\
\text { contenidos y el trabajo } \\
\text { mismo. }\end{array}$ & & & $\begin{array}{l}\text { Favorece el } \\
\text { monitoreo. }\end{array}$ & \\
\hline avorece & & & & \\
\hline
\end{tabular}

Fuente: Elaboración propia, 2013.

Como puede observarse, el estudiantado aduce que la técnica los beneficia en cuanto deben realizar un mayor análisis de la información presentada, complementar con búsqueda de información adicional e integrar conocimientos a situaciones reales o de su futuro quehacer como docentes.

Desde una modalidad a distancia, la técnica también les provee un espacio de autorreflexión y revisión de su propio dominio temático, ya que por ejemplo en el aspecto de "comentarios" del portafolio, es necesaria una justificación teórica para poder realizarlos. Por otra parte, ya que el o la estudiante no pueden contar con una supervisión inmediata de su trabajo (tal y como sucedería en una modalidad regular) si le permite revisar su propio avance, esto por la dinámica de trabajo que se presenta para el portafolio.

Con respecto a los aspectos por mejorar, se obtuvieron menos observaciones en este sentido, ya que únicamente el 17\% de los estudiantes consultados señalaron aspectos por mejorar en relación con la técnica. En este sentido, las respuestas de las y los estudiantes se organizaron en el siguiente cuadro.

\section{CUADRO No 1}

Aspectos por mejorar en la técnica de portafolio como instrumento de evaluación cualitativa en el curso de Detección de Problemas de Aprendizaje de la Universidad Estatal a Distancia

\begin{tabular}{llc} 
& & $\#$ \\
1. & Trabajo muy delicado, minucioso, laborioso y requiere dedicación, compromiso, esfuerzo y tiempo & 29 \\
9. & La calificación de esta técnica es complicada y lenta & 3 \\
10. & Brinda la oportunidad de expresarse de manera creativa, muy distinto a los trabajos dirigidos & 3 \\
2. & Requiere dominio de los contenidos planteados & 2 \\
3. & Conviene dar inducción a quienes son nuevos en su aplicación & 2 \\
4. & Requiere de un acceso adecuado a herramientas tecnológicas para la búsqueda de información & 2 \\
5. & Se convierte en un trabajo extenso, cansado y complicado & 2 \\
7. & Para su aplicación requiere conocimientos previos (elaboración de citas bibliográfica, hacer & 2 \\
& introducción, etc.) & 2 \\
11. & Es exhausta y redundante. & 1 \\
6. Es una técnica compleja, nueva y desconocida & 1 \\
8. Puntaje asignado a la elaboración de la técnica es muy alto. & 1 \\
12. Los temas propuestos encasillan el trabajo & 50 \\
\hline
\end{tabular}

Fuente: Elaboración propia, 2012 
Pese a que fueron pocas las observaciones en el sentido de aspectos negativos,

Los aspectos señalados como negativos o de mejora se circunscriben en algunos casos a situaciones muy específicas de las condiciones del estudiantado, en otros a la dinámica de la técnica misma y al hecho de estar enmarcada en un modelo de educación a distancia; básicamente se podrían rescatar los siguientes aspectos:

1. De la condición de los y las estudiantes:

- el portafolio requiere dedicación, compromiso y esfuerzo;

- resulta un "shock" para estudiantes acostumbrados a una evaluación tradicional.

2. De la técnica:

- requiere de acceso adecuado a herramientas tecnológicas;

- es delicada y minuciosa;

- resulta un trabajo extenso y complicado;

- requiere de adecuados procesos de selección de material e investigación.

3. Para la Cátedra de Psicopedagogía que administra el curso:

- puntaje asignado a esta técnica es muy alto;

- debe darse inducción para su realización.

Desde los aspectos por mejorar, es importante tener claro la dificultad que presentan los y las estudiantes al enfrentarse a nuevas experiencias evaluativas; por ello, sienten la propuesta como extensa, complicada o muy minuciosa; y, efectivamente, si se compara el portafolio con una prueba escrita de ítems específicos como serían selección, única, completar o respuesta breve, el nivel de exigencia en la elaboración del trabajo es mucho mayor.

Ahora bien, el beneficio radicaría en la no propiciación de la memorización para una prueba, sino en la aplicación de los contenidos y la construcción de aprendizaje a partir de dicha aplicación. Además, el estudiantado puede verificar su avance y profundidad del portafolio a partir de las instrucciones que le han sido brindadas, con la posibilidad de construir a partir del error.

\section{Conclusiones}

Explorar nuevas y acordes técnicas de evaluación en un modelo de educación a distancia, constituye un gran reto para quien o quienes ofrecen sus cursos y desean desde la innovación favorecer el aprendizaje significativo.

En ese sentido, el portafolio como técnica de evaluación permite que el estudiantado pueda evidenciar el dominio temático desde una propuesta integradora, que conlleve al análisis, la reflexión y la aplicación de los contenidos desarrollados en el los cursos.

De igual forma para quienes administran los cursos o los desarrollan (hacen labor tutorial), el portafolio ofrece la oportunidad de dar seguimiento real al estudiantado, identificando sus dificultades, evidenciando aprendizajes y construyendo una base teórica que sirva de sustento para modificación y mejora de la técnica.

Desde la perspectiva del estudiantado, la técnica favorece el desarrollo de habilidades de pensamiento, tales como la síntesis, el análisis, la integración y aplicación de contenidos. Por otra parte, implica para el o la estudiante un esfuerzo mayor en áreas de investigación, selección de información y determinación de la pertinencia de la misma.

No se debe perder de vista que este tipo de técnica puede generar resistencia en algunos (as) estudiantes, esto debido a la organización y al tipo de requerimientos para desarrollarla. Teniendo esta consideración es pertinente que quien administre el curso pueda brindar los insumos necesarios para orientar la elaboración del portafolio, de tal forma que la técnica se convierta en un apoyo real al proceso de aprendizaje y no un simple requisito evaluativo del curso.

La técnica en sí misma permite que se ajuste a las necesidades de la población estudiantil, que se varíen las actividades según el énfasis que quiera dársele a un contenido o temática de interés e inclusive que se realicen mejores a partir de los resultados obtenidos en los cursos anteriores. 


\section{Referencias bibliográficas}

Ávila, Patricia. (2005). Calidad en la educación a distancia: Algunas reflexiones. Unidad de Investigación y Modelos Educativos de ILCE. Ecuador.

Barberá, Edebé. (2005)." La evaluación de competencias complejas: la práctica del portafolio". Educere La Revista Venezolana de Educación, vol 9, n 31, pp 5-11.

Barragán, Ricardo. (2005). “ El Portafolio, metodología de evaluación y aprendizaje de cara al nuevo Espacio Europeo de Educación Superior. Una Experiencia práctica en la Universidad de Sevilla". Revista Latinoamericana de Tecnología Educativa, vol 4, n 1, pp 121-139.

Canalejas, María. (2010) "El portafolio como herramienta didáctica: un estudio en escuelas universitarias de enfermería." EDUC MED, vol 13, n 1, pp 53-61.

Danielson, Charlotte. (1997). An Introduction to Using Portfolios in the Classroom. Alexandria, Virginia: Association for Supervision and Curriculum Development.

de la Peña, Maria; Pastor, Maria y Selma, Victoria. (2012). La evaluación de los alumnos a través del Portafolio. REJIE: Revista Jurídica de Investigación e Innovación Educativa. №. 6, pp. 81-94. En: http://www.eumed.net/rev/rejie

Flores, Guadalupe. y Ramírez, Maria. (2009). Interrelación de la evaluación de los aprendizajes con la retroalimentación como estrategia para la mejora educativa. Memorias del XVIII Encuentro Internacional de Educación a Distancia. Universidad de Guadalajara. Mexico

García, Fátima. (2005) "El papel de los portafolios electrónicos en la enseñanza-aprendizaje de las lenguas." Glosas Didácticas. Revista Electrónica Internacional. n 14, pp 112-119.
Hernández Roberto , Fernández Carlos y Baptista Pilar. (2003). Metodología de la investigación. Distrito Federal, México Ed:Mc Graw Hill.

Manrique, Villavicencio. (2004). El aprendizaje autónomo en la educación a distancia. Latin Educa 2004. Primer Congreso Virtual Latinoamericano de Educación a Distancia

Martínez, Silvia. (2007). "Una experiencia de innovación del portafolio del alumno, en diplomatura de educación social, desde el marco de la educación superior en Europa". Educatio Siglo XXI, n 25, pp 125 -144.

Murillo, Gabriela. (2012) "El portafolio como instrumento clave para la evaluación en educación superior". Revista Electrónica Actualidades Investigativas en Educación, vol 12, no 1, pp 1-23 En: http://revista.inie.ucr.ac.cr/

Pozo, María y García, Beatriz. (2006). "El portafolio del alumnado: una investigación -acción en el aula universitaria." Revista de Educación, n³41, pp 737 -756.

UNED (1997). Descripción Curricular de Asignatura para el Curso Detección de Problemas de Aprendizaje. San José, Costa Rica.

UNED (2001). Orientaciones para el Curso Detección de Problemas de Aprendizaje. I Cuatrimestre 2011. San José, Costa Rica.

UNED, 2012. Centro de Operaciones Académicas. Funciones Generales. En: http://estatico.uned.ac.cr/operaciones/ default.shtml

Vera, María y Canalejas, María (2007). "El portafolio como recurso de aprendizaje e instrumento de evaluación de estudiantes repetidores de enfermería". Revista de Educación Médica, vol 10, $\mathrm{n}^{\circ}$ 2, pp 114-120. 
ANEXO 1

Aspectos positivos de la elaboración de la técnica de portafolio

Aspectos positivos de la elaboración de la técnica de portafolio

- Ayuda a planificar, ordenar, seleccionar, recopilar, comparar, discriminar y dar secuencia a los datos de manera sistemática.

- Ayuda a promover el espíritu crítico y analítico.

- Excelente forma de evaluar. Permite relacionar la teoría con la práctica (realidad)

- Fomenta la lectura

- Requiere de información adicional (actualizarse)

- Ayuda a planificar, ordenar, seleccionar, recopilar, comparar, discriminar y dar secuencia a los datos de manera sistemática.

- Ayuda a promover el espíritu crítico y analítico.

- Requiere de parte del estudiante mayor interés y compromiso.

- Permite interiorizar la información y el desarrollo del aprendizaje significativo por ser una técnica más práctica.

- Ayuda a documentar el proceso y ritmo del aprendizaje.

- Permite la investigación y guía sobre el desarrollo y asimilación de los conceptos.

- Fomenta la lectura, ya que se debe leer mucho para obtener la información

- Requiere de información adicional (actualizarse)

- Es una nueva manera de aprender y comprender los temas

- Genera reflexión y análisis durante su elaboración.

- Requiere de información adicional (actualizarse)

- Requiere de parte del estudiante mayor interés y compromiso.

- Con el uso de la técnica los resultados obtenidos son creativos, originales e individuales.

- Permite evaluar tanto al docente y como al alumno, de manera conjunta.

- Se utiliza como manual de orientación sobre lo que se debe realizar

- Es un recurso que funciona como un archivo ya que permite la búsqueda de información.

- Permite ser autocritico con la elaboración del trabajo

- Permite su aplicación en diferentes momentos y niveles del proceso de aprendizaje

- Se adecúa a la forma de trabajo de cada persona. Permite el crecimiento intelectual, el auto aprendizaje y la auto evaluación

- Es una técnica agradable e innovadora

- Esta técnica se convierte en una cadena de conocimiento ya que despierta la motivación y el interés por conocer e investigar otros temas que surgen de la recopilación inicial.

- Permite llevar el control de los procesos y actividades que se realizan con los estudiantes.

- Esta técnica enriquece y potencia el aprendizaje.

- Permite explotar las cualidades y destrezas del aplicador.

- Genera análisis por parte del que elabora la técnica contribuyendo a un aprendizaje significativo

- Promueve el interés en la investigación de otros temas.

- Como técnica evaluativa es más valiosa que un examen porque permite al estudiante "aprender haciendo"

- Permite expresar sentimientos y emociones. En otras palabras involucra emocionalmente al que elabora la técnica.

- Es una técnica funcional, integradora y constructivista.
\# De

respuestas

35

29

27

21

18

16

16

9

9

7

7

5

5

5

5

4

4

4

2

2

2

2

2

2

2

1

1

1

1 
- El producto obtenido (portafolio) se convierte en una herramienta de consulta valiosa para el trabajo del docente.

- Permite enriquecer los contenidos solicitados con nuestra opinión, experiencia y percepción de los contenidos, de manera amplia y abierta.

- Muestra los avances logrados por los estudiantes.

- Despierta la motivación y el interés por investigar otros temas que surgen de la recopilación inicial.

- Es una técnica práctica, vivencial y gradual.

- Fomenta la lectura ya que se debe consultar diversidad de fuentes

- El uso de la técnica de portafolio brinda un valor agregado como lo son las vivencias de las personas observadas o entrevistadas.

- Ayuda a mejorar o reforzar las técnicas de investigación para trabajos futuros.

- Permite crear conclusiones propias

- Genera motivaciones intrínsecas en el individuo, por ejemplo querer constituirse en un agente de cambio en su comunidad

- Mide o evalúa el esfuerzo y dedicación que haya puesto el estudiante en su trabajo

- Sirve de monitoreo

- Se aprende y se enriquece de los diferentes puntos de vista de los autores consultados

- Permite tomar una posición personal respecto a los distintos temas desarrollados.

- Permite la acumulación y consolidación del aprendizaje

- Requiere que el estudiante documente adecuadamente los temas.

- Permite aprender o interiorizar con mayor rapidez el tema que se evalúa.

Genera orden en el desarrollo de los temas

Fuente: Elaboración propia, 2013. 
- 